\title{
Generalized $S$-space-forms with two structure vector fields
}

\author{
Alfonso Carriazo, Luis M. Fernández and Ana M. Fuentes* \\ (Communicated by P. Eberlein)
}

\begin{abstract}
We introduce and study generalized $S$-space-forms with two structure vector fields. We also present several examples of these manifolds such as certain hypersurfaces of Sasakianspace-forms, principal toroidal bundles and warped products. Moreover, we investigate generalized $S$-space-forms endowed with an additional structure and we obtain some obstructions for them to be $S$-manifolds.
\end{abstract}

Key words. Metric $f$-manifold, $f$-contact manifold, $f$ - $K$-contact manifold, $S$-manifold, generalized $S$-space-form.

2000 Mathematics Subject Classification. 53C25, 53C40

\section{Introduction}

It is an interesting problem to analyze what kind of Riemannian manifolds may be determined by special pointwise expressions for their curvatures. For instance, it is well known that the sectional curvatures of a Riemannian manifold determine the curvature tensor field completely. So, if $(M, g)$ is a connected Riemannian manifold with dimension greater than 2 and its curvature tensor field $R$ has the pointwise expression

$$
R(X, Y) Z=\lambda\{g(X, Z) Y-g(Y, Z) X\}
$$

where $\lambda$ is a differentiable function on $M$, then $M$ is a space of constant sectional curvature, that is, a real-space-form and $\lambda$ is a constant function.

Further, when the manifold is equipped with some additional structure, it is sometimes possible to obtain conclusions from the special form of the curvature tensor field for this structure too. Thus, an almost-Hermitian manifold $(M, J, g)$ is said to be a generalized

* The first two authors are partially supported by the PAI project (Junta de Andalucía, Spain, 2009) and by the MEC-FEDER grant MTM 2007-61284 (MEC, Spain, 2007). 
complex-space-form [13] if its curvature tensor satisfies

$$
\begin{aligned}
R(X, Y) Z= & f_{1}\{g(Y, Z) X-g(X, Z) Y\} \\
& +f_{2}\{g(X, J Z) J Y-g(Y, J Z) J X+2 g(X, J Y) J Z\}
\end{aligned}
$$

where $f_{1}$ and $f_{2}$ are differentiable functions on $M$. This name derives from the fact that, when $M$ is a complex-space-form, that is, a Kählerian manifold of constant holomorphic curvature equal to $c$, the curvature tensor field of $M$ satisfies (1.1) with $f_{1}=f_{2}=c / 4$. Moreover, in [13], F. Tricerri and L. Vanhecke proved that any connected generalized complex-space-form of dimension greater than or equal to 6 and with $f_{2}$ not identically zero is a complex-space-form (in particular, $f_{1}=f_{2}$ are constant functions). For the 4-dimensional case, Z. Olszak [11] characterized generalized complex-space-forms with non-constant function $f_{2}$, giving some non-trivial examples.

Since Sasakian-spaces-forms play a similar role in contact metric geometry to that of complex-space-forms in complex geometry, P. Alegre, D.E. Blair and A. Carriazo have defined and studied generalized Sasakian-space-forms [1] as those almost-contact metric manifolds $(M, \varphi, \xi, \eta, g)$ whose curvature tensor field satisfies

$$
\begin{aligned}
& R(X, Y) Z=f_{1}\{g(Y, Z) X-g(X, Z) Y\} \\
& \quad+f_{2}\{g(X, \varphi Z) \varphi Y-g(Y, \varphi Z) \varphi X+2 g(X, \varphi Y) \varphi Z\} \\
& \quad+f_{3}\{\eta(X) \eta(Z) Y-\eta(Y) \eta(Z) X+g(X, Z) \eta(Y) \xi-g(Y, Z) \eta(X) \xi\}
\end{aligned}
$$

$f_{1}, f_{2}, f_{3}$ being differentiable functions on $M$. If $M$ is actually a Sasakian-space-form, that is a Sasakian manifold with constant $\varphi$-sectional curvature equal to $c$, then:

$$
f_{1}=\frac{c+3}{4} ; \quad f_{2}=f_{3}=\frac{c-1}{4} .
$$

More in general, K. Yano [14] introduced the notion of $f$-structure on a $(2 m+s)$ dimensional manifold as a tensor field $f$ of type $(1,1)$ and rank $2 m$ satisfying $f^{3}+f=0$. Almost complex $(s=0)$ and almost contact $(s=1)$ structures are well-known examples of $f$-structures. In this context, D.E. Blair [2] defined $K$-manifolds (and particular cases of $S$-manifolds and $C$-manifolds) as the analogue of Kählerian manifolds in the almost complex geometry and of quasi-Sasakian manifolds in the almost contact geometry and he showed that the curvature of either $S$-manifolds or $C$-manifolds is completely determined by their $f$-sectional curvatures. Later, M. Kobayashi and S. Tsuchiya [10] got expressions of the curvature tensor field of $S$-manifolds and $C$-manifolds when their $f$-sectional curvature is constant depending on such a constant.

For these reasons, we consider that it is interesting to introduce a notion of generalized $S$-space-form on metric $f$-manifolds (see Section 2 for a precise definition of these manifolds) and we have limited our research to the case $s=2$ which appeared in the study of hypersurfaces in almost contact manifolds $[3,8]$. To this end, we have followed the same procedure as in almost complex and almost contact cases, that is, we have substituted the constants in the expression of the curvature tensor field of an $S$-space-form (an $S$-manifold of constant $f$-sectional curvature) obtained in [10] by certain differentiable functions on the manifold. So, $S$-space-forms are natural examples of generalized 
$S$-space-forms. Furthermore, we check that $C$-space-forms are also generalized $S$-spaceforms.

We have organized the paper in the following way. In Section 2 we review definitions and formulas concerning metric $f$-manifolds which we shall use later. In Section 3 we introduce the notion of generalized $S$-space-form and we give some non-trivial examples. So, we prove that pseudo-umbilical, totally contact-umbilical, totally contact-geodesic, totally umbilical and totally geodesic hypersurfaces of a generalized Sasakian-space-form are generalized $S$-space-forms. Moreover, the bundle space of a principal toroidal bundle over a Kählerian manifold and the warped product of $\mathbb{R}$ times a generalized Sasakianspace-form are generalized $S$-space-forms too. Finally, in Section 4 we study generalized $S$-space-forms endowed with an additional structure and the relationships between the functions in such a case. Thus, we prove that any generalized $S$-space-form with a metric $f$ - $K$-contact structure is actually an $S$-manifold. The same result holds for a metric $f$-contact structure with some additional conditions on the functions. We also obtain an obstruction for a generalized $S$-space-form to be an $S$-manifold, depending on the functions.

\section{Metric $f$-manifolds}

A $(2 m+s)$-dimensional Riemannian manifold $(M, g)$ endowed with an $f$-structure $f$ (that is, a tensor field of type $(1,1)$ and rank $2 m$ satisfying $f^{3}+f=0$ [14]) is said to be a metric $f$-manifold if, moreover, there exist $s$ global vector fields $\xi_{1}, \ldots, \xi_{s}$ on $M$ (called structure vector fields) such that, if $\eta_{1}, \ldots, \eta_{s}$ are the dual 1 -forms of $\xi_{1}, \ldots, \xi_{s}$, then

$$
\begin{gathered}
f \xi_{\alpha}=0 ; \quad \eta_{\alpha} \circ f=0 ; \quad f^{2}=-I+\sum_{\alpha=1}^{s} \eta_{\alpha} \otimes \xi_{\alpha} ; \\
g(X, Y)=g(f X, f Y)+\sum_{\alpha=1}^{s} \eta_{\alpha}(X) \eta_{\alpha}(Y),
\end{gathered}
$$

for any $X, Y \in \mathcal{X}(M)$ and $\alpha=1, \ldots, s$. The distribution on $M$ spanned by the structure vector fields is denoted by $\mathcal{M}$ and its complementary orthogonal distribution is denoted by $\mathcal{L}$. Consequently, $T M=\mathcal{L} \oplus \mathcal{M}$. Moreover, if $X \in \mathcal{L}$, then $\eta_{\alpha}(X)=0$, for any $\alpha=1, \ldots, s$, and if $X \in \mathcal{M}$, then $f X=0$.

If the structure vector fields of a metric $f$-manifold are Killing vector fields, then

$$
\mathrm{d} \eta_{\alpha}(X, Y)=\left(\nabla_{X} \eta_{\alpha}\right) Y=g\left(\nabla_{X} \xi_{\alpha}, Y\right),
$$

for any $X, Y \in \mathcal{X}(M)$ and $\alpha=1, \ldots, s$, where $\nabla$ is denoting the Riemannian connection of the manifold. The curvature tensor field $R$ of a metric $f$-manifold with Killing structure vector fields satisfies [6]

$$
R\left(\xi_{\alpha}, X, \xi_{\beta}, Y\right)=Y \eta_{\alpha}\left(\nabla_{X} \xi_{\alpha}\right)-g\left(\nabla_{X} \xi_{\beta}, \nabla_{Y} \xi_{\alpha}\right),
$$

for any $X, Y \in \mathcal{X}(M)$ and $\alpha, \beta=1, \ldots, s$. 
Let $F$ be the 2-form on $M$ defined by $F(X, Y)=g(X, f Y)$, for any $X, Y \in \mathcal{X}(M)$. Since $f$ is of rank $2 m$, we have

$$
\eta_{1} \wedge \cdots \wedge \eta_{s} \wedge F^{m} \neq 0
$$

and, particularly, $M$ is orientable. A metric $f$-manifold is said to be a metric $f$-contact manifold if $F=\mathrm{d} \eta_{\alpha}$, for any $\alpha=1, \ldots, s$. On the other hand, a metric $f$-contact manifold it is said to be a metric $f$ - $K$-contact manifold if the structure vector fields are Killing vector fields. When $s=1$, metric $f$-contact manifolds correspond to contact manifolds and metric $f$ - $K$-contact manifolds to $K$-contact manifolds. Furthermore, in a metric $f$ - $K$-contact manifold, from (2.2) it easy to show that:

$$
\nabla_{X} \xi_{\alpha}=-f X, \quad X \in \mathcal{X}(M), \quad \alpha=1, \ldots, s .
$$

For the curvature tensor field $R$ of a metric $f$ - $K$-contact manifold it is known that [6]

$$
R\left(X, \xi_{\alpha}\right) Y=-\left(\nabla_{X} f\right) Y
$$

for any $X, Y \in \mathcal{X}(M)$ and $\alpha=1, \ldots, s$.

The $f$-structure $f$ is said to be normal if

$$
[f, f]+2 \sum_{\alpha=1}^{s} \xi_{\alpha} \otimes d \eta_{\alpha}=0
$$

where $[f, f]$ denotes the Nijenhuis tensor of $f$.

A metric $f$-manifold is said to be a $K$-manifold [2] if it is normal and d $F=0$. In a $K$-manifold $M$, the structure vector fields are Killing vector fields [2]. Then:

$$
\nabla_{\xi_{\alpha}} \xi_{\beta}=0, \quad \alpha, \beta=1, \ldots, s .
$$

A $K$-manifold is called an $S$-manifold if $F=\mathrm{d} \eta_{\alpha}$, for any $\alpha$ (that is, if it is a metric $f$ - $K$-contact manifold) and a $C$-manifold if $\mathrm{d} \eta_{\alpha}=0$, for any $\alpha$. Note that, for $s=0$, a $K$-manifold is a Kählerian manifold and, for $s=1$, a $K$-manifold is a quasi-Sasakian manifold, an $S$-manifold is a Sasakian manifold and a $C$-manifold is a cosymplectic manifold. When $s \geq 2$, non-trivial examples can be found in [2,9]. Moreover, a $K$-manifold $M$ is an $S$-manifold if and only if

$$
\nabla_{X} \xi_{\alpha}=-f X, \quad X \in \mathcal{X}(M), \quad \alpha=1, \ldots, s,
$$

and it is a $C$-manifold if and only if

$$
\nabla_{X} \xi_{\alpha}=0, \quad X \in \mathcal{X}(M), \quad \alpha=1, \ldots, s .
$$

It is easy to show that any $f-K$-contact manifold $M$ is an $S$-manifold if and only if

$$
\left(\nabla_{X} f\right) Y=\sum_{\alpha}\left\{g(f X, f Y) \xi_{\alpha}+\eta_{\alpha}(Y) f^{2} X\right\}
$$

for any $X, Y \in \mathcal{X}(M)$. 
On the other hand, the curvature tensor field $R$ of a $K$-manifold $M$ satisfies [6]

$$
R\left(\xi_{\alpha}, X, \xi_{\beta}, Y\right)=-g\left(\nabla_{X} \xi_{\beta}, \nabla_{Y} \xi_{\alpha}\right),
$$

for any $X, Y \in \mathcal{X}(M)$ and $\alpha, \beta=1, \ldots, s$. If $M$ is an $S$-manifold, then [6]

$$
R(X, Y) \xi_{\alpha}=\sum_{\beta=1}^{s}\left\{\eta_{\beta}(X) f^{2} Y-\eta_{\beta}(Y) f^{2} X\right\}
$$

for any $X, Y \in \mathcal{X}(M)$ and $\alpha=1, \ldots, s$.

A plane section $\pi$ on a metric $f$-manifold $M$ is said to be an $f$-section if it is determined by a unit vector $X \in \mathcal{L}_{x}$ and $f X$. The sectional curvature of $\pi$ is called an $f$-sectional curvature. An $S$-manifold (respectively, a $C$-manifold) is said to be an $S$ space-form (respectively, a $C$-space-form) if it has constant $f$-sectional curvature $c$ and then, it is denoted by $M(c)$. In such cases, the curvature tensor field $R$ of $M(c)$ satisfies [10]:

$$
\begin{aligned}
& R(X, Y, Z, W)=\sum_{\alpha, \beta}\left(g(f X, f W) \eta_{\alpha}(Y) \eta_{\beta}(Z)-g(f X, f Z) \eta_{\alpha}(Y) \eta_{\beta}(W)\right. \\
& \left.\quad+g(f Y, f Z) \eta_{\alpha}(X) \eta_{\beta}(W)-g(f Y, f W) \eta_{\alpha}(X) \eta_{\beta}(Z)\right) \\
& \quad+\frac{c+3 s}{4}(g(f X, f W) g(f Y, f Z)-g(f X, f Z) g(f Y, f W)) \\
& \quad+\frac{c-s}{4}(F(X, W) F(Y, Z)-F(X, Z) F(Y, W)-2 F(X, Y) F(Z, W))
\end{aligned}
$$

respectively

$$
\begin{array}{r}
R(X, Y, Z, W)=\frac{c}{4}(g(f X, f W) g(f Y, f Z)-g(f X, f Z) g(f Y, f W) \\
\quad+F(X, W) F(Y, Z)-F(X, Z) F(Y, W)-2 F(X, Y) F(Z, W))
\end{array}
$$

for any $X, Y, Z, W \in \mathcal{X}(M)$.

\section{Generalized $S$-space-forms with two structure vector fields}

From now on, we only consider metric $f$-manifolds with two structure vector fields $\xi_{1}$ and $\xi_{2}$. Then, we say that such a metric $f$-manifold

$$
\left(M, f, \xi_{1}, \xi_{2}, \eta_{1}, \eta_{2}, g\right)
$$


is a generalized $S$-space-form if there exists differentiable functions $F_{1}, \ldots, F_{8}$ on $M$ such that the curvature tensor field of $M$ satisfies

$$
\begin{aligned}
& R(X, Y) Z=F_{1}\{g(Y, Z) X-g(X, Z) Y\} \\
& \quad+F_{2}\{g(X, f Z) f Y-g(Y, f Z) f X+2 g(X, f Y) f Z\} \\
& \quad+F_{3}\left\{\eta_{1}(X) \eta_{1}(Z) Y-\eta_{1}(Y) \eta_{1}(Z) X+g(X, Z) \eta_{1}(Y) \xi_{1}-g(Y, Z) \eta_{1}(X) \xi_{1}\right\} \\
& \quad+F_{4}\left\{\eta_{2}(X) \eta_{2}(Z) Y-\eta_{2}(Y) \eta_{2}(Z) X+g(X, Z) \eta_{2}(Y) \xi_{2}-g(Y, Z) \eta_{2}(X) \xi_{2}\right\} \\
& \quad+F_{5}\left\{\eta_{1}(X) \eta_{2}(Z) Y-\eta_{1}(Y) \eta_{2}(Z) X+g(X, Z) \eta_{1}(Y) \xi_{2}-g(Y, Z) \eta_{1}(X) \xi_{2}\right\} \\
& \quad+F_{6}\left\{\eta_{2}(X) \eta_{1}(Z) Y-\eta_{2}(Y) \eta_{1}(Z) X+g(X, Z) \eta_{2}(Y) \xi_{1}-g(Y, Z) \eta_{2}(X) \xi_{1}\right\} \\
& \quad+F_{7}\left\{\eta_{1}(X) \eta_{2}(Y) \eta_{2}(Z) \xi_{1}-\eta_{2}(X) \eta_{1}(Y) \eta_{2}(Z) \xi_{1}\right\} \\
& \quad+F_{8}\left\{\eta_{2}(X) \eta_{1}(Y) \eta_{1}(Z) \xi_{2}-\eta_{1}(X) \eta_{2}(Y) \eta_{1}(Z) \xi_{2}\right\},
\end{aligned}
$$

for any $X, Y, Z \in \mathcal{X}(M)$.

This kind of manifold appears as a natural generalization of $S$-space-forms because a straightforward computation from (2.11) gives that any $S$-space-form $M(c)$ with two structure vector fields is a generalized $S$-space-form with functions:

$$
F_{1}=\frac{c+6}{4} ; \quad F_{2}=F_{7}=F_{8}=\frac{c-2}{4} ; \quad F_{3}=F_{4}=\frac{c+2}{4} ; \quad F_{5}=F_{6}=-1 .
$$

Moreover, any $C$-space-form $M(c)$ with two structure vector fields is also a generalized $S$-space-form. In fact, from (2.12), we only have to take:

$$
F_{1}=F_{2}=F_{3}=F_{4}=F_{7}=F_{8}=\frac{c}{4} ; \quad F_{5}=F_{6}=0 .
$$

On the other hand, from (3.1) it is easy to compute the $f$-sectional curvature of $M$ :

Proposition 3.1. The $f$-sectional curvature of a generalized $S$-space-form $M=(M, f$, $\left.\xi_{1}, \xi_{2}, \eta_{1}, \eta_{2}, g\right)$ is equal to $F_{1}+3 F_{2}$.

Now, we are going to present other non-trivial examples:

Example 3.1. Let $(\widetilde{M}, \varphi, \xi, \eta, g)$ be a generalized Sasakian-space-form [1] with functions $f_{1}, f_{2}$ and $f_{3}$ and let $M$ be an isometrically immersed (orientable) hypersurface of $\widetilde{M}$ such that the vector field $\xi$ is always tangent to $M$. If $N$ denotes the unit normal vector field of $M$ in $\widetilde{M}$ and we put

$$
\begin{gathered}
\xi_{1}=\xi ; \quad \xi_{2}=-\varphi N ; \\
\eta_{1}=\eta ; \quad \eta_{2}(X)=-g(X, \varphi N) ; \\
f X=\varphi X-\eta_{2}(X) N,
\end{gathered}
$$

for any vector field $X$ tangent to $M$, then $\left(M, f, \xi_{1}, \xi_{2}, \eta_{1}, \eta_{2}, g\right)$ is a metric $f$-manifold [15]. Moreover, the 2-form $F$ of $M$ satisfies

$$
F(X, Y)=g(X, \varphi Y), \quad \text { for } X, Y \text { tangent to } M .
$$


Now, if we denote by $R$ the curvature tensor field of $M$ and by $A$ the shape operator $A_{N}$, then, by using the well-known Gauss equation for the curvature tensor fields, from (1.2) we easily get

$$
\begin{aligned}
& R(X, Y) Z=f_{1}\{g(Y, Z) X-g(X, Z) Y\} \\
& \quad+f_{2}\{g(X, f Z) f Y-g(Y, f Z) f X+2 g(X, f Y) f Z\} \\
& \quad+f_{3}\left\{\eta_{1}(X) \eta_{1}(Z) Y-\eta_{1}(Y) \eta_{1}(Z) X\right. \\
& \left.\quad+g(X, Z) \eta_{1}(Y) \xi_{1}-g(Y, Z) \eta_{1}(X) \xi_{1}\right\}+g(A Y, Z) A X-g(A X, Z) A Y,
\end{aligned}
$$

for any $X, Y, Z \in T M$, where we are also denoting by $f_{1}, f_{2}$ and $f_{3}$ the corresponding restrictions of such functions to $M$.

Next, suppose that $M$ is a pseudo-umbilical hypersurface of $\widetilde{M}$, that is, the shape operator $A$ satisfies (see [15])

$$
A X=\widetilde{f}_{1}\left(X-\eta_{1}(X) \xi_{1}\right)+\widetilde{f}_{2} \eta_{2}(X) \xi_{2}-\eta_{1}(X) \xi_{2}-\eta_{2}(X) \xi_{1},
$$

for any $X$ tangent to $M, \widetilde{f}_{1}$ and $\widetilde{f}_{2}$ being differentiable functions on $M$. Thus, a direct computation from (3.2) gives that $M$ is a generalized $S$-space-form. In fact:

$$
\begin{gathered}
F_{1}=f_{1}+\widetilde{f}_{1}^{2} ; \quad F_{2}=f_{2} ; \quad F_{3}=f_{2}+\widetilde{f}_{1}^{2} ; \quad F_{4}=-\widetilde{f}_{1} \tilde{f}_{2} ; \\
F_{5}=F_{6}=\widetilde{f}_{1} ; \quad F_{7}=F_{8}=-1-\widetilde{f}_{1} \tilde{f}_{2} .
\end{gathered}
$$

In particular, totally contact-umbilical $\left(\widetilde{f}_{1} \equiv 1, \widetilde{f}_{2} \equiv 0\right)$ and, respectively, totally contact-geodesic $\left(\widetilde{f}_{1}=\widetilde{f}_{2} \equiv 0\right)$ hypersurfaces of a generalized Sasakian-space-form are generalized $S$-space-forms. Moreover, any totally umbilical $(A X=\lambda X$, for any $X$ tangent to $M$ ) hypersurface of a Sasakian space-form is a generalized $S$-space-form, with

$$
F_{1}=f_{1}+\lambda^{2} ; \quad F_{2}=f_{2} ; \quad F_{3}=f_{3} ; \quad F_{4}=F_{5}=F_{6}=F_{7}=F_{8}=0
$$

and any totally geodesic $(A X=0$, for any $X \in T M)$ hypersurface of a Sasakian spaceform is a generalized $S$-space-form, with:

$$
F_{1}=f_{1} ; \quad F_{2}=f_{2} ; \quad F_{3}=f_{3} ; \quad F_{4}=F_{5}=F_{6}=F_{7}=F_{8}=0 .
$$

Example 3.2. Let $M=\left(M, f, \xi_{1}, \xi_{2}, \eta_{1}, \eta_{2}, g\right)$ be an $S$-manifold which is the bundle space of a principal toroidal bundle over a Kählerian manifold $N=(N, J, G)$, with dimensions $2 n+2$ and $2 n$, respectively. We denote by $\pi: M \longrightarrow N$ the projection map which is a Riemannian submersion. Then, it is known (see [2,4] for more details) that
(i) $\quad \nu_{x}=\operatorname{span}\left\{\xi_{1_{x}}, \xi_{2_{x}}\right\}$;
(ii) $(J X)^{*}=f X^{*}$
(iii) $G(X, Y)=g\left(X^{*}, Y^{*}\right)$,

for any point $x \in M$ and any vector fields $X, Y$ on $N$, where $\nu_{x}$ is the vertical subspace at $x$ and ${ }^{*}$ is denoting the horizontal lift. Let $R$ and $\widetilde{R}$ be the curvature tensor fields of $M$ and $N$, respectively. Then, in [7] it is proved that

$$
\begin{aligned}
(\widetilde{R}(X, Y) Z)^{*}= & R\left(X^{*}, Y^{*}\right) Z^{*}+2\left\{g\left(X^{*}, f Z^{*}\right) f Y^{*}\right. \\
& \left.-g\left(Y^{*}, f Z^{*}\right) f X^{*}+2 g\left(X^{*}, f Y^{*}\right) f Z^{*}\right\},
\end{aligned}
$$


for any $X, Y, Z$ tangent to $N$. In these conditions, if $N$ is a generalized complex-spaceform with functions $f_{1}$ and $f_{2}$, then from (1.1) we obtain that

$$
\begin{aligned}
& R\left(X^{*}, Y^{*}\right) Z^{*}=\left(f_{1} \circ \pi\right)\left\{g\left(Y^{*}, Z^{*}\right) X^{*}-g\left(X^{*}, Z^{*}\right) Y^{*}\right\} \\
& \quad+\left(f_{2} \circ \pi-2\right)\left\{g\left(X^{*}, f Z^{*}\right) f Y^{*}-g\left(Y^{*}, f Z^{*}\right) f X^{*}+2 g\left(X^{*}, f Y^{*}\right) f Z^{*}\right\}
\end{aligned}
$$

for any $X, Y, Z$ tangent to $N$. Since we can write

$$
X=\widetilde{X}+\sum_{\alpha=1}^{2} \eta_{\alpha}(X) \xi_{\alpha},
$$

for any vector field $X$ tangent to $M$, where $\widetilde{X}$ is an horizontal vector field and taking into account that any $S$-manifold is a metric $f-K$-contact manifold too, a straightforward computation using (2.5), (2.8), (2.10) and (3.3) gives that $M$ is a generalized $S$-spaceform, with functions:

$$
\begin{gathered}
F_{1}=f_{1} \circ \pi ; \quad F_{2}=f_{2} \circ \pi-2 ; \quad F_{3}=F_{4}=f_{1} \circ \pi-1 ; \\
F_{5}=F_{6}=-1 ; \quad F_{7}=F_{8}=f_{1} \circ \pi-2 .
\end{gathered}
$$

Example 3.3. Let $(\widetilde{M}, \varphi, \xi, \eta, \widetilde{g})$ be a generalized Sasakian space-form with functions $f_{1}, f_{2}$ and $f_{3}$. Then, its curvature tensor field $\widetilde{R}$ satisfies (1.2). Consider now a warped product (see [12]) $M=\mathbb{R} \times{ }_{h} \widetilde{M}$, where $h>0$ is a differentiable function on $\mathbb{R}$. Then, the warped product metric on $M$ is given by

$$
g_{h}=\pi_{1}^{*}\left(g_{\mathbb{R}}\right)+\left(h \circ \pi_{1}\right)^{2} \pi_{2}^{*}(\widetilde{g}),
$$

where $\pi_{1}$ and $\pi_{2}$ are the projections from $\mathbb{R} \times \widetilde{M}$ on $\mathbb{R}$ and $\widetilde{M}$, respectively. Given any vector field $X$ on $M$, written as

$$
X=\left(a \frac{\mathrm{d}}{\mathrm{d} t}, \widetilde{X}\right), \quad \widetilde{X} \text { on } \widetilde{M},
$$

with $a$ any differentiable function on $\mathbb{R}$ and $t$ the coordinate of $\mathbb{R}$, we define an $f$-structure on $M$ by:

$$
f X=(0, \varphi \widetilde{X})=\left(\varphi\left(\pi_{2}\right)_{*} X\right)^{*} .
$$

Taking

$$
\begin{gathered}
\xi_{1}=\left(0, \frac{1}{h} \xi\right), \quad \xi_{2}=\left(\frac{\mathrm{d}}{\mathrm{d} t}, 0\right), \\
\eta_{1}\left(\left(a \frac{\mathrm{d}}{\mathrm{d} t}, \widetilde{X}\right)\right)=h \eta(\widetilde{X}), \quad e_{2}\left(\left(a \frac{\mathrm{d}}{\mathrm{d} t}, \widetilde{x}\right)\right)=a,
\end{gathered}
$$

we easily show that $\left(M, f, \xi_{1}, \xi_{2}, \eta_{1}, e_{2}, g_{h}\right)$ is a metric $f$-manifold. Moreover, we have that, for any $X$ on $M$ :

$$
X=\left(a \frac{\mathrm{d}}{\mathrm{d} t}, \tilde{X}\right)=\eta_{2}(X) \xi_{2}+(0, \tilde{X}) .
$$


Now, by using (1.2) and the results of O'Neill [12] concerning the Riemannian connections and curvature tensor fields of warped products (see Lemmas 4.6 and 4.7 of [1]), a straightforward calculation proves that $M$ is a generalized $S$-space-form with functions:

$$
\begin{gathered}
F_{1}=\frac{\left(f_{1} \circ \pi_{2}\right)-h^{\prime 2}}{h^{2}} ; \quad F_{2}=\frac{f_{2} \circ \pi_{2}}{h^{2}} ; \\
F_{3}=F_{7}=F_{8}=\frac{f_{3} \circ \pi_{2}}{h^{2}} ; \quad F_{4}=\frac{\left(f_{1} \circ \pi_{2}\right)-h^{\prime 2}}{h^{2}}+\frac{h^{\prime \prime}}{h} ; \\
F_{5}=F_{6}=0 .
\end{gathered}
$$

Consequently, new examples of generalized $S$-space-forms can be obtained from the examples of generalized Sasakian-space-forms given in [1]. In particular, if $N(c)$ is a complex-space-form of constant holomorphic curvature $c$, then $M=\mathbb{R} \times{ }_{h_{2}}\left(\mathbb{R} \times \times_{h_{1}} N(c)\right)$ is a generalized $S$-space-form $\left(h_{1}, h_{2}>0\right.$ differentiable functions on $\left.\mathbb{R}\right)$ with functions:

$$
\begin{gathered}
F_{1}=\frac{c-4\left(h_{1}^{\prime}\right)^{2}-4 h_{1}^{2}\left(h_{2}^{\prime}\right)^{2}}{4 h_{1}^{2} h_{2}^{2}} ; \quad F_{2}=\frac{c}{4 h_{1}^{2} h_{2}^{2}} ; \\
F_{3}=F_{7}=F_{8}=\frac{c-4\left(h_{1}^{\prime}\right)^{2}+4 h_{1} h_{1}^{\prime \prime}}{4 h_{1}^{2} h_{2}^{2}} ; \\
F_{4}=\frac{c-4\left(h_{1}^{\prime}\right)^{2}-4 h_{1}^{2}\left(h_{2}^{\prime}\right)^{2}+4 h_{1}^{2} h_{2} h_{2}^{\prime \prime}}{4 h_{1}^{2} h_{2}^{2}} \\
F_{5}=F_{6}=0 .
\end{gathered}
$$

On the other hand and as particular case too, the usual Riemannian product (a warped product with $h \equiv 1$ ) of $\mathbb{R}$ and a generalized Sasakian-space-form with functions $f_{1}, f_{2}$ and $f_{3}$ is a generalized $S$-space-form with functions:

$$
\begin{gathered}
F_{1}=F_{4}=f_{1} \circ \pi_{2} ; \quad F_{2}=f_{2} \circ \pi_{2} ; \quad F_{3}=F_{7}=F_{8}=f_{3} \circ \pi_{2} ; \\
F_{5}=F_{6}=0 .
\end{gathered}
$$

\section{Structure of generalized $S$-space-forms}

In this section we investigate generalized $S$-space-forms $M$ with two structure vector fields endowed with an additional structure and we study the relationships between the functions in such a case. First and for later use, we write two particular expressions of (3.1):

$$
\begin{aligned}
R\left(X, \xi_{1}\right) Y= & F_{1}\left\{\eta_{1}(Y) X-g(X, Y) \xi_{1}\right\} \\
& +F_{3}\left\{\eta_{1}(X) \eta_{1}(Y) \xi_{1}-\eta_{1}(Y) X+g(X, Y) \xi_{1}-\eta_{1}(X) \eta_{1}(Y) \xi_{1}\right\} \\
& +F_{4}\left\{\eta_{2}(X) \eta_{2}(Y) \xi_{1}-\eta_{2}(X) \eta_{1}(Y) \xi_{2}\right\}+ \\
& +F_{5}\left\{\eta_{1}(X) \eta_{2}(Y) \xi_{1}-\eta_{2}(Y) X+g(X, Y) \xi_{2}-\eta_{1}(X) \eta_{1}(Y) \xi_{2}\right\} \\
& +F_{7}\left\{-\eta_{2}(X) \eta_{2}(Y) \xi_{1}\right\}+F_{8}\left\{\eta_{2}(X) \eta_{1}(Y) \xi_{2}\right\}
\end{aligned}
$$


and

$$
\begin{aligned}
R\left(X, \xi_{2}\right) Y= & F_{1}\left\{\eta_{2}(Y) X-g(X, Y) \xi_{2}\right\} \\
& +F_{3}\left\{\eta_{1}(X) \eta_{1}(Y) \xi_{2}-\eta_{1}(X) \eta_{2}(Y) \xi_{1}\right\} \\
& +F_{4}\left\{\eta_{2}(X) \eta_{2}(Y) \xi_{2}-\eta_{2}(Y) X+g(X, Y) \xi_{2}-\eta_{2}(X) \eta_{2}(Y) \xi_{2}\right\} \\
& +F_{6}\left\{\eta_{2}(X) \eta_{1}(Y) \xi_{2}-\eta_{1}(Y) X+g(X, Y) \xi_{1}-\eta_{2}(X) \eta_{2}(Y) \xi_{1}\right\} \\
& +F_{7}\left\{\eta_{1}(X) \eta_{2}(Y) \xi_{1}\right\}+F_{8}\left\{-\eta_{1}(X) \eta_{1}(Y) \xi_{2}\right\},
\end{aligned}
$$

for any $X, Y \in \mathcal{X}(M)$. Now, we can prove the following theorem; its proof is based on Bianchi's second identity,

$$
\begin{aligned}
g\left(\left(\mathfrak{S}_{X, Y, Z}(\nabla R)(X, Y, Z)\right) W, V\right)= & g\left(\left(\nabla_{X} R\right)(Y, Z) W, V\right)+g\left(\left(\nabla_{Y} R\right)(Z, X) W, V\right) \\
& +g\left(\left(\nabla_{Z} R\right)(X, Y) W, V\right)=0,
\end{aligned}
$$

for any vector fields $X, Y, Z, W, V$ tangent to $M$, together with the definition of a generalized $S$-space-form (3.1).

Theorem 4.1. Let $M=\left(M, f, \xi_{1}, \xi_{2}, \eta_{1}, \eta_{2}, g\right)$ be a generalized $S$-space-form with $F_{2}$ not identically zero and $\operatorname{dim}(M) \geq 6$. Suppose that the following conditions hold on $M$ :

(a) $\nabla_{X} \xi_{\alpha} \in \mathcal{L}$, for any $X \in \mathcal{X}(M)$ and any $\alpha=1,2$;

(b) $g\left(\nabla_{X} \xi_{\alpha}, X\right)=0$, for any $X \in \mathcal{L}$ and any $\alpha=1,2$.

Then:

(i) $\nabla_{\xi_{\beta}} \xi_{\alpha}=0$, for any $\alpha, \beta=1,2$ and $F_{7}=F_{8}$.

(ii) If there are two unit vector fields $X, Y \in \mathcal{L}$ such that $\mathrm{d} \eta_{1}(X, Y) \neq 0\left(\right.$ or $\mathrm{d} \eta_{2}(X, Y)$ $\neq 0$ ), then $F_{5}=F_{6}$.

(iii) If either $F_{2}=F_{3}$ or $F_{2}=F_{4}$, then $F_{1}$ and $F_{2}$ are constant functions. Moreover, if $M$ is a K-manifold, then $F_{1}-F_{2} \geq 0$ and, in this case, if $F_{1}=F_{2}=F_{3}=F_{4}$, then $M$ is a C-manifold.

Proof. Let $X \in \mathcal{L}$ be a unit vector field. Then, choosing another unit vector field $Y \in \mathcal{L}$ such that $g(X, Y)=g(X, f Y)=0$ (which is possible since $\operatorname{dim}(M) \geq 6$ ) and putting $Z=\xi_{\alpha}, W=\xi_{\beta}(\alpha, \beta=1,2)$ and $V=f X$, from (4.3) and (3.1) we obtain:

$$
F_{2} g\left(\nabla_{\xi_{\alpha}} \xi_{\beta}, f Y\right)=0, \quad \alpha, \beta=1,2 .
$$

But, from the hypothesis, $F_{2} \neq 0$ and $\nabla_{\xi_{a}} \xi_{\beta} \in \mathcal{L}$, for $\alpha, \beta=1,2$ and so, (4.4) implies that:

$$
\nabla_{\xi_{\alpha}} \xi_{\beta}=0, \quad \alpha, \beta=1,2 .
$$

Consequently, $R\left(\xi_{1}, \xi_{2}, \xi_{1}, \xi_{2}\right)=0$ and, from (4.1), $F_{1}-F_{3}-F_{4}+F_{8}=0$. Moreover, $R\left(\xi_{2}, \xi_{1}, \xi_{2}, \xi_{1}\right)=0$ and, from (4.2), $F_{1}-F_{3}-F_{4}+F_{7}=0$. Thus, $F_{7}=F_{8}$ and we have proved (i).

Next, applying Bianchi's second identity (4.3) together with (3.1) and (4.5),

$$
\begin{aligned}
& g\left(\left(\mathfrak{S}_{X, \xi_{2}, \xi_{1}}(\nabla R)\left(X, \xi_{2}, \xi_{1}\right)\right) \xi_{1}, V\right)=\left(\xi_{1} F_{6}-\xi_{2} F_{3}\right) g(X, V) \\
& \quad+F_{4} g\left(\nabla_{X} \xi_{2}, V\right)+F_{6} g\left(\nabla_{X} \xi_{1}, V\right)-F_{8} g\left(\nabla_{X} \xi_{2}, V\right)=0
\end{aligned}
$$


for any unit vector fields $X, V \in \mathcal{L}$. Putting $V=X$, we have that $\left(\xi_{1} F_{6}-\xi_{2} F_{3}\right)=0$ and so:

$$
F_{4} g\left(\nabla_{X} \xi_{2}, V\right)+F_{6} g\left(\nabla_{X} \xi_{1}, V\right)-F_{8} g\left(\nabla_{X} \xi_{2}, V\right)=0,
$$

for any unit vector field $V \in \mathcal{L}$.

Now, from (4.3), (3.1) and (4.5) again

$$
\begin{aligned}
& g\left(\left(\mathfrak{S}_{X, Y, \xi_{1}}(\nabla R)\left(X, Y, \xi_{1}\right)\right) \xi_{1}, \xi_{2}\right)=F_{4}\left\{g\left(\nabla_{Y} \xi_{2}, X\right)-g\left(\nabla_{X} \xi_{2}, Y\right)\right\} \\
& \quad+F_{5}\left\{g\left(\nabla_{Y} \xi_{1}, X\right)-g\left(\nabla_{X} \xi_{1}, Y\right)\right\}+F_{8}\left\{g\left(\nabla_{X} \xi_{2}, Y\right)-g\left(\nabla_{Y} \xi_{2}, X\right)\right\}=0
\end{aligned}
$$

for any unit vector fields $X, Y \in \mathcal{L}$. Thus, from (4.7) and (4.8) we have that

$$
\begin{aligned}
0 & =\left(F_{5}-F_{6}\right)\left\{g\left(\nabla_{Y} \xi_{1}, X\right)-g\left(\nabla_{X} \xi_{1}, Y\right)\right\} \\
& =\left(F_{5}-F_{6}\right) \eta_{1}([X, Y])=-2\left(F_{5}-F_{6}\right) \mathrm{d} \eta_{1}(X, Y)
\end{aligned}
$$

and so, taking the vector fields of the hypothesis of (ii), $F_{5}=F_{6}$. Reasoning similarly in the case where $\mathrm{d} \eta_{2}(X, Y) \neq 0$, we complete the proof of (ii).

From now on, we consider two unit vector fields $X, Y \in \mathcal{L}$ such that $g(X, Y)=$ $g(X, f Y)=0$, which exist since $\operatorname{dim}(M) \geq 6$. Then, putting $Z=f Y$ and $W=X$ into (4.3), from (3.1) we obtain that, for $V=f X$ and $V=f Y$, respectively,

$$
Y F_{1}-3 F_{2} g\left(\left(\nabla_{X} f\right) X, f Y\right)=0
$$

and:

$$
2 X F_{2}-3 F_{2} g\left(\left(\nabla_{Y} f\right) X, f Y\right)-3 F_{2} g\left(\left(\nabla_{f Y} f\right) Y, X\right)=0 .
$$

Interchanging the roles of $X$ and $Y$ in (4.9), we have:

$$
X F_{1}-3 F_{2} g\left(\left(\nabla_{Y} f\right) Y, f X\right)=0 .
$$

But, since $g(f X, Y)=0$, (4.11) implies that $X F_{1}=3 F_{2} g\left(\left(\nabla_{f Y} f\right) f Y, f X\right)=$ $-3 F_{2} g\left(\left(\nabla_{f Y} f\right) Y, X\right)$ and, then, from (4.10) and (4.11), we get, for any $X \in \mathcal{L}$ :

$$
X\left(F_{1}+F_{2}\right)=0 .
$$

Next, we apply Bianchi's second identity (4.3) again when $Z=\xi_{\alpha}(\alpha=1,2)$, $W=X$ and $V=Y$. By using (3.1), we obtain:

$$
\xi_{\alpha} F_{1}=0, \alpha=1,2 .
$$

Similarly, putting $W=f X$ and $V=f Y$, we have:

$$
\xi_{\alpha} F_{2}=0, \alpha=1,2 .
$$

On the other hand, Bianchi's second identity again, together with (3.1), gives, for $Z=W=\xi_{1}$ and $V=Y$,

$$
X\left(F_{1}-F_{3}\right)=0
$$


and, for $Z=W=\xi_{2}$ and $V=Y$ :

$$
X\left(F_{1}-F_{4}\right)=0
$$

In these conditions, if $F_{2}=F_{3}$, from (4.12), (4.13), (4.14) and (4.15) we show that $F_{1}$ and $F_{2}$ are constant functions. If $F_{2}=F_{4}$, we do the same using (4.16).

Finally, we suppose that $M$ is a $K$-manifold. Then, from (3.1), if $F_{2}=F_{3}$, we get $R\left(X, \xi_{1}\right) \xi_{1}=\left(F_{1}-F_{2}\right) X$ and so, from (2.9), $F_{1}-F_{2}=\left\|\nabla_{X} \xi_{1}\right\|^{2} \geq 0$. If $F_{2}=F_{4}$, we obtain the same result by using $\xi_{2}$ instead of $\xi_{1}$. Therefore, from (2.7) we deduce that, if $F_{1}-F_{2}=0$ and $F_{2}=F_{3}=F_{4}$, then $M$ is a $C$-manifold.

Observe that conditions (a) and (b) are natural because, for instance, either metric $f$ - $K$-contact manifolds (in particular, $S$-manifolds) or $C$-manifolds fulfill them. Furthermore, the hypothesis of (ii) holds, for example, for metric $f$ - $K$-contact manifolds too. On the other hand, the above theorem can be considered as the version for generalized $S$ space-forms of Theorem 12.7 of [13] for generalized complex-space-forms and of either the main theorem in [5] or Theorem 3.4 in [1] for generalized Sasakian-space-forms.

Now, we are interested in generalized $S$-space-forms with a general function $F_{2}$. First, we have:

Proposition 4.1. Let $M=\left(M, f, \xi_{1}, \xi_{2}, \eta_{1}, \eta_{2}, g\right)$ be a generalized $S$-space-form. If $M$ is either a metric $f$ - $K$-contact manifold or a $K$-manifold, then:

$$
F_{1}+F_{7}=F_{3}+F_{4}=F_{1}+F_{8} .
$$

Consequently, in these cases, $F_{7}=F_{8}$.

Proof. If $M$ is a metric $f$ - $K$-contact manifold, from (2.3) and (2.4) we have $R\left(\xi_{1}, \xi_{2}, \xi_{1}\right.$, $\left.\xi_{2}\right)=0$ and then, by using (4.1), $F_{1}-F_{3}-F_{4}+F_{8}=0$. Similarly, from $R\left(\xi_{2}, \xi_{1}, \xi_{2}, \xi_{1}\right)=$ 0 and (4.2), we get $F_{1}-F_{3}-F_{4}+F_{7}=0$.

The proof is the same if $M$ is a $K$-manifold by using (2.6) and (2.9).

From Proposition 4.1, we deduce that totally umbilical (respectively, totally geodesic) hypersurfaces of a $K$-contact generalized Sasakian-space-form with functions $f_{1}=f_{3}+1$ and $f_{2}$ (see Proposition 3.6 of [1]) are generalized $S$-space-forms which are neither metric $f$ - $K$-contact manifolds nor $K$-manifolds because (see Example 3.1)

$$
\left.F_{1}+F_{7}=f_{1}+\lambda^{2} \neq F_{3}+F_{4}=f_{3} \quad \text { (respectively, } F_{1}+F_{7}=f_{1} \neq F_{3}+F_{4}=f_{3}\right) .
$$

On the other hand, from (4.17) again, we also deduce that the warped product $\mathbb{R} \times{ }_{h} \widetilde{M}$, where $\widetilde{M}$ is a generalized Sasakian-space-form (see Example 3.3), is a generalized $S$ space-form which can be neither a metric $f$ - $K$-contact manifold nor a $K$-manifold, unless $h^{\prime \prime}=0$.

Moreover, we have the following proposition. 
Proposition 4.2. Let $M=\left(M, f, \xi_{1}, \xi_{2}, \eta_{1}, \eta_{2}, g\right)$ be a generalized $S$-space-form. If $M$ is a metric $f$-K-contact manifold, then:

$$
F_{1}-F_{3}=F_{1}-F_{4}=1 ; \quad F_{5}=F_{6}=-1 .
$$

Consequently:

$$
F_{3}=F_{4}=1+F_{7} ; \quad F_{1}-F_{7}=2 .
$$

Proof. From (2.3) and (2.4) we obtain $R\left(\xi_{\alpha}, X, \xi_{\beta}, X\right)=-1$, for any unit vector field $X \in \mathcal{L}$ and any $\alpha, \beta=1,2$; then, by using (4.1), (4.2) and (4.17) we complete the proof.

In particular, as any $S$-manifold is a metric $f-K$-contact manifold, (4.18) and (4.19) hold on $S$-manifolds. In this situation, we can prove:

Theorem 4.2. Any generalized $S$-space-form $M=\left(M, f, \xi_{1}, \xi_{2}, \eta_{1}, \eta_{2}, g\right)$ with a metric $f$-K-contact structure is an $S$-manifold.

Proof. From (4.1) and by using $F_{7}=F_{8}$, we obtain:

$$
\begin{aligned}
& R\left(X, \xi_{1}\right) Y=\left(F_{1}-F_{3}\right)\left(\eta_{1}(Y) X-g(X, Y) \xi_{1}\right) \\
& \quad+\left(F_{4}-F_{7}\right)\left(\eta_{2}(X) \eta_{2}(Y) \xi_{1}-\eta_{2}(X) \eta_{1}(Y) \xi_{2}\right) \\
& \quad+F_{5}\left(\eta_{1}(X) \eta_{2}(Y) \xi_{1}-\eta_{2}(Y) X+g(X, Y) \xi_{2}-\eta_{1}(X) \eta_{1}(Y) \xi_{2}\right) .
\end{aligned}
$$

Thus, substituting (4.18) and (4.19) into (4.20) and taking into account (2.5), we get Formula (2.8) and, consequently, $M$ is an $S$-manifold.

With respect to metric $f$-contact manifolds, we have the following result.

Theorem 4.3. Any generalized $S$-space-form $M=\left(M, f, \xi_{1}, \xi_{2}, \eta_{1}, \eta_{2}, g\right)$ of dimension $2 n+2$ with a metric $f$-contact structure and such that $F_{3}=F_{4}, F_{7}=F_{8}$ and $F_{1}-F_{3}=$ $F_{4}-F_{7}=1$ is an $S$-manifold.

Proof. First, from (4.1), (4.2) and the hypothesis, we deduce $K\left(\xi_{1}, \xi_{2}\right)=0$, where $K$ is denoting the sectional curvature. Moreover, a direct computation by using (4.1) and (4.2) again shows that $S\left(\xi_{1}, \xi_{1}\right)=2 n\left(F_{1}-F_{3}\right)=2 n$ and $S\left(\xi_{2}, \xi_{2}\right)=2 n\left(F_{1}-F_{4}\right)=2 n$, $S$ being the Ricci curvature tensor. Then, by using Theorem 3.8 of [6], we obtain that $\xi_{1}$ and $\xi_{2}$ are Killing vector fields, that is, $M$ is a metric $f-K$-contact manifold and, so, from Theorem 4.2, it is an $S$-manifold.

On the other hand, for generalized $S$-space-forms with a $K$-structure, we can prove the following proposition.

Proposition 4.3. Let $M=\left(M, f, \xi_{1}, \xi_{2}, \eta_{1}, \eta_{2}, g\right)$ be a generalized $S$-space-form. If $M$ is a K-manifold, then:

$$
F_{1}-F_{3}, F_{1}-F_{4}, F_{3}-F_{7}, F_{4}-F_{7} \geq 0 ; \quad F_{5}=F_{6} .
$$

In particular, if $M$ is a $C$-manifold, then:

$$
F_{1}=F_{3}=F_{4}=F_{7}=F_{8} ; \quad F_{5}=F_{6}=0 .
$$


Proof. From (2.9), (4.1) and (4.2), we obtain

$$
\begin{gathered}
\left\|\nabla_{X} \xi_{1}\right\|^{2}=F_{1}-F_{3} ; \quad\left\|\nabla_{X} \xi_{2}\right\|^{2}=F_{1}-F_{4} ; \\
g\left(\nabla_{X} \xi_{1}, \nabla_{X} \xi_{2}\right)=-F_{5}=-F_{6},
\end{gathered}
$$

for any unit vector field $X \in \mathcal{L}$. Then, the proof is completed by using Proposition 4.1. For $C$-manifolds, we only have to consider (2.7).

Finally, we want to obtain some conditions for the function $F_{2}$ in the case of generalized $S$-space-forms endowed with an $S$-structure. First, by using (2.8), a direct computation gives:

Lemma 4.1. Let $M=\left(M, f, \xi_{1}, \xi_{2}, \eta_{1}, \eta_{2}, g\right)$ be a generalized $S$-space-form. If $M$ is an $S$-manifold, then

$$
\begin{aligned}
R(X, Y, Z, f W) & +R(X, Y, f Z, W)=2\{g(Y, Z) F(X, W)-g(X, Z) F(Y, W) \\
& +g(X, W) F(Y, Z)-g(Y, W) F(X, Z)\}
\end{aligned}
$$

for any $X, Y, Z, W \in \mathcal{L}$.

Hence, we obtain the following theorem.

Theorem 4.4. Let $M=\left(M, f, \xi_{1}, \xi_{2}, \eta_{1}, \eta_{2}, g\right)$ be a generalized $S$-space-form. If $M$ is an $S$-manifold, then $F_{1}-F_{2}=2$ and, consequently:

$$
F_{2}=F_{7}=F_{8}=F_{3}-1=F_{4}-1 .
$$

Proof. From (3.1),

$$
\begin{aligned}
R(X, Y, Z, f W) & +R(X, Y, f Z, W)=\left(F_{1}-F_{2}\right)\{g(Y, Z) F(X, W) \\
& -g(X, Z) F(Y, W)+g(X, W) F(Y, Z)-g(Y, W) F(X, Z)\},
\end{aligned}
$$

for any $X, Y, Z, W \in \mathcal{L}$. So, $F_{1}-F_{2}=2$ follows from (4.23). The use of (4.18) and (4.19) completes the proof.

As a consequence of the above theorem, if $M$ is the bundle space of a principal toroidal bundle over a Kählerian generalized complex-space-form $N$ with functions $f_{1}$ and $f_{2}$ (see Example 3.2), then, since $F_{1}-F_{2}=2$, we see that $f_{1} \circ \pi=f_{2} \circ \pi$ which implies $f_{1}=f_{2}$ because $\pi$ is onto. This result was known if $n \geq 3$ [13].

\section{References}

[1] P. Alegre, D. E. Blair, A. Carriazo, Generalized Sasakian-space-forms. Israel J. Math. 141 (2004), 157-183. MR2063031 (2005f:53057) Zbl 1064.53026

[2] D. E. Blair, Geometry of manifolds with structural group $\mathcal{U}(n) \times \mathcal{O}(s)$. J. Differential Geometry 4 (1970), 155-167. MR0267501 (42 \#2403) Zbl 0202.20903 
[3] D. E. Blair, G. D. Ludden, Hypersurfaces in almost contact manifolds. Tôhoku Math. J. (2) 21 (1969), 354-362. MR0251668 (40 \#4895)

[4] D. E. Blair, G. D. Ludden, K. Yano, Differential geometric structures on principal toroidal bundles. Trans. Amer. Math. Soc. 181 (1973), 175-184. MR0319099 (47 \#7645) Zbl 0276.53026

[5] P. Bueken, L. Vanhecke, Curvature characterizations in contact geometry. Riv. Mat. Univ. Parma (4) 14 (1988), 303-313 (1989). MR1007089 (90f:53063) Zbl 0689.53021

[6] J. L. Cabrerizo, L. M. Fernández, M. Fernández, The curvature tensor fields on $f$-manifolds with complemented frames. An. Ştiinţ. Univ. Al. I. Cuza Iaşi Secţ. I a Mat. 36 (1990), 151-161. MR1109808 (92d:53031) Zbl 0737.53031

[7] L. M. Fernández, Submanifolds in toroidal bundles. In: Proceedings of the Sixth International Colloquium on Differential Geometry (Santiago de Compostela, 1988), volume 61 of Cursos Congr. Univ. Santiago de Compostela, 85-98, Univ. Santiago de Compostela, Santiago de Compostela 1989. MR1040839 (90m:53068) Zbl 0737.53031

[8] S. I. Goldberg, K. Yano, Globally framed f-manifolds. Illinois J. Math. 15 (1971), 456-474. MR0278247 (43 \#3978) Zbl 0215.23002

[9] I. Hasegawa, Y. Okuyama, T. Abe, On $p$ th Sasakian manifolds. J. Hokkaido Univ. Ed. Sect. II A 37 (1986), 1-16. MR880988 (88e:53047)

[10] M. Kobayashi, S. Tsuchiya, Invariant submanifolds of an $f$-manifold with complemented frames. Kōdai Math. Sem. Rep. 24 (1972), 430-450. MR0319103 (47 \#7649) Zbl 0246.53038

[11] Z. Olszak, On the existence of generalized complex space forms. Israel J. Math. 65 (1989), 214-218. MR998671 (90c:53091) Zbl 0674.53061

[12] B. O'Neill, Semi-Riemannian geometry, volume 103 of Pure and Applied Mathematics. Academic Press 1983. MR719023 (85f:53002) Zbl 0531.53051

[13] F. Tricerri, L. Vanhecke, Curvature tensors on almost Hermitian manifolds. Trans. Amer. Math. Soc. 267 (1981), 365-397. MR626479 (82j:53071) Zbl 0484.53014

[14] K. Yano, On a structure defined by a tensor field $f$ of type $(1,1)$ satisfying $f^{3}+f=0$. Tensor (N.S.) 14 (1963), 99-109. MR0159296 (28 \#2513) Zbl 0122.40705

[15] K. Yano, M. Kon, Generic submanifolds of Sasakian manifolds. Kodai Math. J. 3 (1980), 163-196. MR588453 (82a:53051) Zbl 0452.53034

Received 16 November, 2007; revised 18 January, 2008

A. Carriazo, L. M. Fernández, A. M. Fuentes, Departmento de Geometría y Topología, Facultad de Matemáticas, Universidad de Sevilla, Apartado de Correos 1160, 41080 Sevilla, Spain Email: carriazo@us.es,lmfer@us.es 